\title{
Final Report of the Japan Neurotrauma Data Bank Project 1998-2001: 1,002 Cases of Traumatic Brain Injury
}

\author{
Norio NAKAMURA*, Akira YAMAURA*, Minoru ShIGEMORI*, \\ Takeki OGAWA*,**, Takashi TOKUTOMI*, Jun-ichi ONO*, \\ Tatsurou KAWAMATA*, and Tetsuya SAKAMOTO* \\ *Japan Neurotrauma Data Bank (JNTDB) Committee (Japan Society of Neurotraumatology, \\ The Japanese Council of Traffic Science); ** Department of Emergency Medicine, \\ The Jikei University School of Medicine, Tokyo
}

\begin{abstract}
A 4-year study (Japan Neurotrauma Data Bank) of the medical treatment of 1002 cases of traumatic brain injuries in Japan was conducted from 1998 to 2001 at 10 emergency medical centers. Patients with severe head injury were eligible for entry with a Glasgow Coma Scale score of 8 or less at admission. Patients who underwent craniotomy were also included. Children under 5 years old were excluded. An original data sheet with 392 items from multi-focal viewpoints, such as etiology of injury, pre-hospital care, initial treatment including neuro-intensive care unit, and surgical treatment, was created. The results show that the patient's age and mechanism of injury are the most important factors in the outcome.
\end{abstract}

Key words: severe brain injury, databank, epidemiology, trauma

\section{Introduction}

The Japan Neurotrauma Data Bank (JNTDB) Committee was founded within the Japan Society of Neurotraumatology in 1997. The committee carried out joint clinical studies (the first pilot study) with 10 medical institutions nationwide to evaluate traumatic brain injuries in Japan. ${ }^{5)}$ The institutions were chosen for excellence and past achievements at their emergency medical centers, especially for the treatment of traumatic brain injuries. Details of the final reports from the 10 institutions covering the years between 1998 and 2001 were outlined.6)

The Traumatic Coma Data Bank (TCDB) in the U.S.A. achieved impressive study results which provided the impetus for our research. ${ }^{3,4)}$ In Japan, the chief cause of head injuries is motor vehicle accidents, and traumatic brain injury is thought to be the most important determinant of the outcome in patients with multiple injuries. However, statistics include little information from medical facilities and comprehensive data that describes situations surrounding traffic deaths or severe head injury from a medical point of view is not available. The

Received January 25, 2006; Accepted August 8, 2006
National Police Agency and the Ministry of Health, Labor and Welfare have data on traffic accidents and deaths by traffic accidents. Furthermore, the automobile industry is conducting research on prevention of accidents. All these organizations are working separately from each other. Therefore, the medical side pertaining to treatment, and the non-medical side pertaining to prevention, should conduct a joint study of head injuries. In order to conduct a comparative study together with other countries, a collection of data relating to multiple aspects of head injuries is essential. Moreover, this study will help improve emergency facilities, medical rehabilitation, and the study of outcome.

\section{Purpose}

The purpose was to evaluate the present status of treatment for traumatic brain injuries as well as to research the factors that affect the outcome of such injuries in Japan. The data sheet was designed for the participating institutions to enter itemized information. Items within this data sheet were chosen for future clinical epidemiological discussions to be held among medical and non-medical organizations including the Ministry of Health, Labor, and Welfare, the National Police Agency, and 
Table 1 Distribution of data by clinical institutions and year

\begin{tabular}{|c|c|c|c|c|c|c|c|c|}
\hline Institution & \multicolumn{2}{|c|}{1998} & \multicolumn{2}{|c|}{1999} & \multicolumn{2}{|c|}{2000} & 2001 & Total \\
\hline B & 10 & 13 & 18 & 15 & 12 & 18 & 26 & 112 \\
\hline $\mathrm{C}$ & 16 & 14 & 17 & 14 & 11 & 8 & 0 & 80 \\
\hline $\mathrm{D}$ & 0 & 16 & 28 & 30 & 40 & 30 & 54 & 198 \\
\hline $\mathrm{G}$ & 11 & 10 & 6 & 9 & 8 & 6 & 8 & 58 \\
\hline $\mathrm{H}$ & 12 & 15 & 21 & 6 & 20 & 15 & 31 & 120 \\
\hline I & 24 & 18 & 15 & 18 & 36 & 27 & 58 & 196 \\
\hline $\mathrm{J}$ & 6 & 10 & 8 & 5 & 6 & 6 & 9 & 50 \\
\hline Total & 115 & 145 & 153 & 131 & 161 & 139 & 256 & 1100 \\
\hline
\end{tabular}

1,002 of 1,100 cases were evaluated as eligible data.

engineers. This collection of such data from the medical side is expected to lead improved treatment, and the advancement of research.

\section{Method}

\section{Subjects}

The population consisted of patients over 6 years old, with consciousness level of Glasgow Coma Scale (GCS) score 8 or less on admission or during follow up (this includes cases of cardiopulmonary arrest on arrival [CPAOA] thought to be caused by traumatic brain injury), and who underwent craniotomy.

\section{Medical institutions}

The following 10 institutions participated, which all have an emergency medical center with a neurosurgeon who can supervise diagnosis, treatment, and management of traumatic brain injuries: Department of Neurosurgery, Sendai City Hospital, Sendai, Miyagi; Department of Neurosurgery, Chiba Emergency Medical Center, Chiba; Department of Neurosurgery, National Disaster Medical Center, Tachikawa, Tokyo; Department of Emergency and Critical Care Medicine, Showa University Hospital, Tokyo; Department of Emergency and Critical Care Medicine, Showa General Hospital, Kodaira, Tokyo; Department of Neurosurgery, Nihon University School of Medicine, Tokyo; Department of Neurosurgery, St. Marianna University School of Medicine, Kawasaki, Kanagawa; Department of Neurosurgery, Saiseikai Shiga Hospital, Ritto, Shiga; Department of Neurosurgery, Kurume University School of Medicine, Kurume, Fukuoka; and Department of Neurosurgery, Japanese Red Cross
Kumamoto Hospital, Kumamoto.

The JNTDB Committee (Japan Society of Neurotraumatology, The Japanese Council of Traffic Sciences) consisted of members of the participating medical institutions, representative delegates, planning delegates, managing delegates, and observers.

\section{Data sheet}

Attending physicians from the participating institutions recorded 392 items onto a data sheet, and accumulated data were sent every 6 months to the secretariat. Computed tomography (CT) and magnetic resonance imaging findings were recorded as written data. CT findings were classified according to the TCDB categories. We incorporated the Abbreviated Injury Scale 1985 (AIS-85) for the evaluation of multiple injuries. The Glasgow Outcome Scale was used to evaluate outcome.

\section{Duration of time}

During the 4 years between 1998 and 2001, data was collected from registered institutions, and after the managing committee checked the items on the collected data, the most updated database was distributed to all committee members. Analysis and statistical evaluation was left to the individual member's method of appraisal. Members met twice a year to report on progress and discussed what was needed for future research.

\section{Results}

\section{Case numbers}

Of the 1,100 accumulated cases, 98 cases (approximately $10 \%$ ), which contained either missing or erroneous information, were omitted to avoid biased 


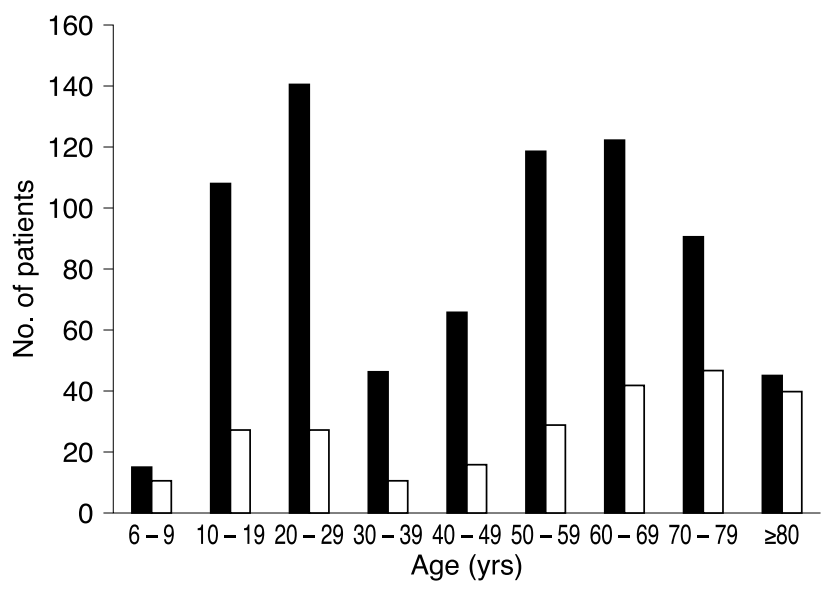

Fig. 1 Distribution of patient age and $\operatorname{sex}(n=$ 1,002). Males were predominant in all age groups. Distribution of patient age showed two peaks between 10 and 29 years and between 50 and 79 years. $\square$ : $m a l e(n=$ 753), $\square$ : female $(n=249)$.

interpretation. Therefore, 1,002 cases which satisfied the eligibility criteria were analyzed. The number of cases amounted to 230-300 each year. Looking at the annual number of cases, one institution reported nine cases and another reported 70 , indicating a wide variation in the number of cases among the respective institutions (mean 28 cases/ institution/year) (Table 1).

\section{Sex and age}

The population contained more males in total, 753 males $(75 \%)$ as compared to 249 females (25\%), with ages ranging from 6 to 98 years (mean $48 \pm 24$ years). There were a higher number of male patients in the 10's, 20's, 50's, 60's, and 70's groups. Among females, the numbers were higher in the 60's to 80's groups. Males and females showed similar numbers in the 80's group (Fig. 1).

\section{GCS on admission}

There were 832 patients ( $83 \%$ ) with GCS score 8 or under on admission. The most common was GCS score 3 in 274 patients but these cases included CPAOA (115 cases) (Fig. 2). Patients were transferred to hospitals within 1 hour in $58 \%$ of cases, and $90 \%$ were transferred within 6 hours.

The findings showed that evaluation of GCS was more appropriate at 48 hours after a patient is admitted to the hospital instead at the time of arrival, because of the occurrence of talk and deteriorate or concussion cases, as collecting data from unstable conditions means consistent changes

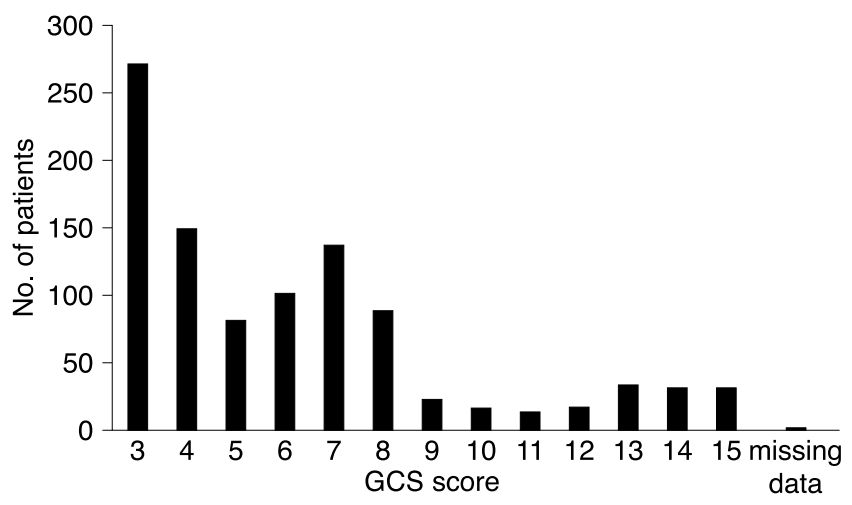

Fig. 2 Glasgow Coma Scale (GCS) on admission (n = 1,002). $832(83 \%)$ of patients had GCS score of 8 or less. 274 patients had GCS score of 3 (including 115 cases of cardiopulmonary arrest on arrival).

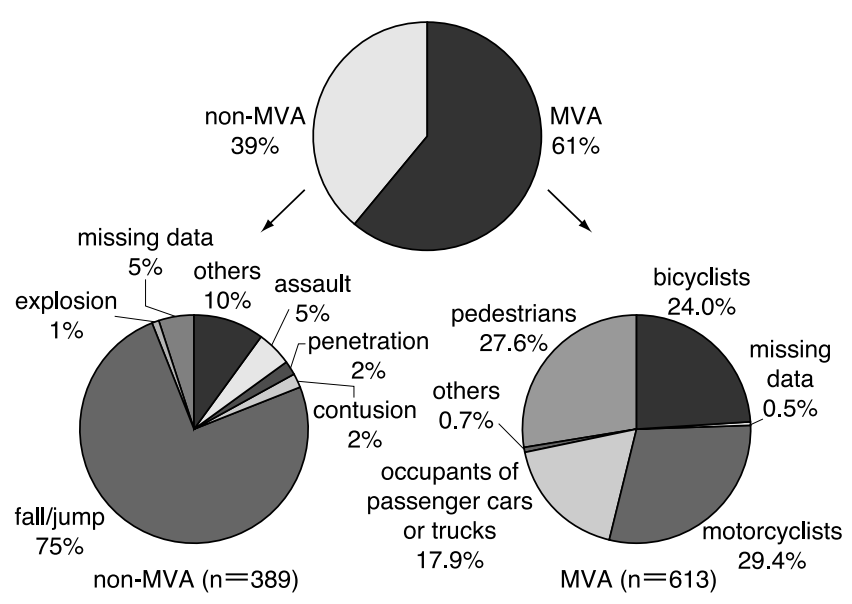

Fig. 3 Cause of injury/accident $(n=1,002)$. Pedestrians, bicyclists, and motorcyclists accounted for most victims of motor vehicle accident (MVA).

are difficult to predict and evaluate.

\section{Cause of injury}

Injuries from motor vehicle accidents accounted for $61 \%$ (613 cases). Non-motor vehicle accidents accounted for $39 \%$ (389 cases). Motor vehicle accidents included motorcyclists, pedestrians, bicyclists, and occupants of passenger cars, trucks, and motorcycles. A large percentage $(75 \%)$ of non-motor vehicle accidents involved falls and jumps (Fig. 3).

\section{Deaths}

There were 525 cases of death (52\%). A significant number of deaths related to motor vehicle accidents 


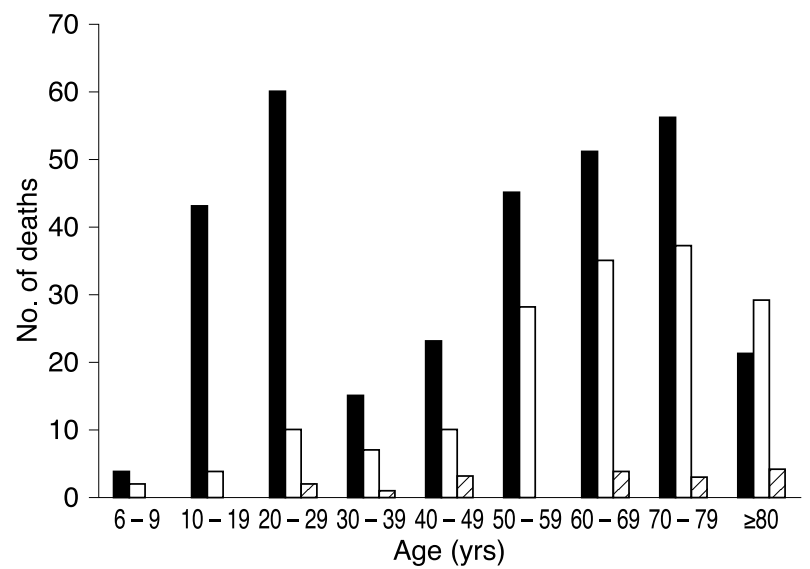

Fig. 4 Number of deaths by age in motor vehicle accident (MVA) and falls/jumps $(n=525)$. MVA was the most frequent cause of death in patients between 10 and 29 years of age. MVA and fall or jump were the two major causes of death in patients between 50 and 79 years of age. $\square$ : MVA, $\square$ : falls/jumps, $Z Z Z A$ : others.

were seen in patients in their 20's, 60's, and 70's. The number of deaths caused by jumps or falls was highest in the 50's to 80's groups. Furthermore, deaths caused by jumps or falls outnumbered deaths related to motor vehicle accidents in patients in the 80's group (Fig. 4).

\section{CT categories}

Classification of traumatic brain injury still remains controversial, because of the complexity in analyzing image data and clinical pathology. TCDB defines two classifications: diffuse brain injury (DBI) and focal brain injury (FBI). However, some cases cannot be distinctly classified as either DBI or FBI. Therefore, in Japan a third category was created for cases in which both DBI and FBI were recognized.

In total, $39 \%$ were categorized as DBI and FBI, $38 \%$ were $\mathrm{FBI}$, and $22 \%$ were DBI. Of injuries caused by motor vehicle accidents (613 cases), $44 \%$ were DBI and FBI, $29 \%$ were DBI, and $26 \%$ were FBI. Of injuries caused by non-motor vehicle accidents (389 cases), $57 \%$ were FBI, $33 \%$ were FBI and DBI, and $10 \%$ were DBI (Fig. 5).

\section{Outcome at time of discharge}

Follow-up assessment of patients was difficult because patients were often transferred to other hospitals/institutions for further treatment. Therefore, outcome was assessed at the time of discharge. Death (D) occurred in $51 \%$ of the cases, vegetative

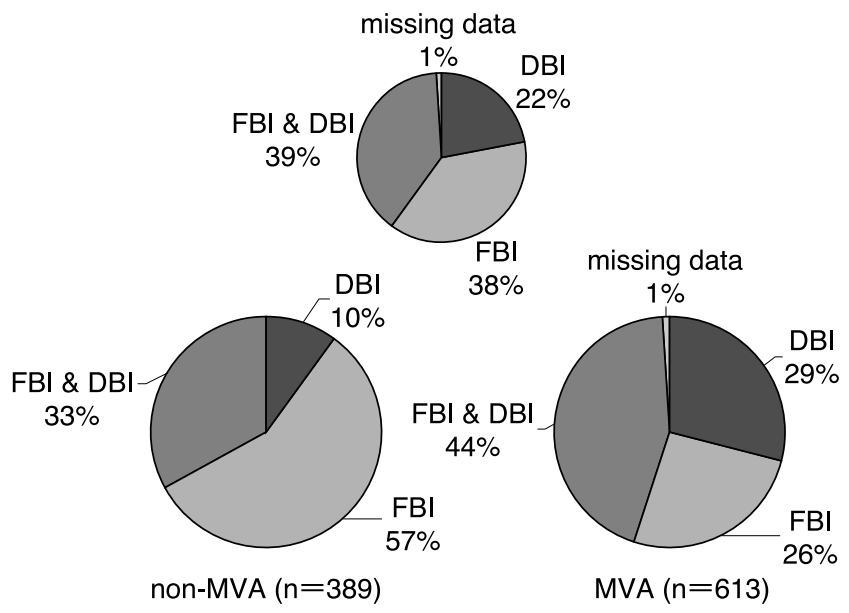

Fig. 5 Focal brain injury (FBI) and diffuse brain injury (DBI) $(n=1,002)$. DBI and $D B I$ associated with FBI accounted for $73 \%$ of cases in the motor vehicle accident (MVA) group, but FBI accounted for $57 \%$ of cases in the non-MVA group.

state in $6 \%$, severe disability in $15 \%$, moderate disability in $13 \%$, and good recovery (GR) in $14 \%$.

\section{Outcome by age}

Figure 6 displays an overview of the outcome according to sex and age. Numbers of both D and GR were significantly high in young males. On the other hand, numbers of D were high but GR was very low in both males and females of around 56 years of age. Outcome for below 49 years of age revealed D $42 \%$ and GR $35 \%$, and above 50 years of age revealed D $61 \%$ and GR 10\% (Fig. 7). Presumably traumatic head injuries undergo worse progression after about 50 years of age. The outcome of traumatic brain injuries clearly changes near age 50 years.

\section{Outcome of multiple injuries}

Multiple injuries were defined as damage of more than AIS-85 score 3 to organs other than the head and/or neck. There were 315 cases of multiple injuries $(31 \%)$. In one study, the patients were divided into below 49 years of age and above 50 years of age. The below 49 years group had D 58\% and GR 17\% (Fig. 8). In another study, the patients were divided into below 69 years old and over 70 years old. The below 69 years group had D 62\% and GR 13\%. The above 70 years group showed a significant increase of $82 \% \mathrm{D}$ and $7 \% \mathrm{GR}$.

\section{Discussion}

Cases for this study were limited to severe head 

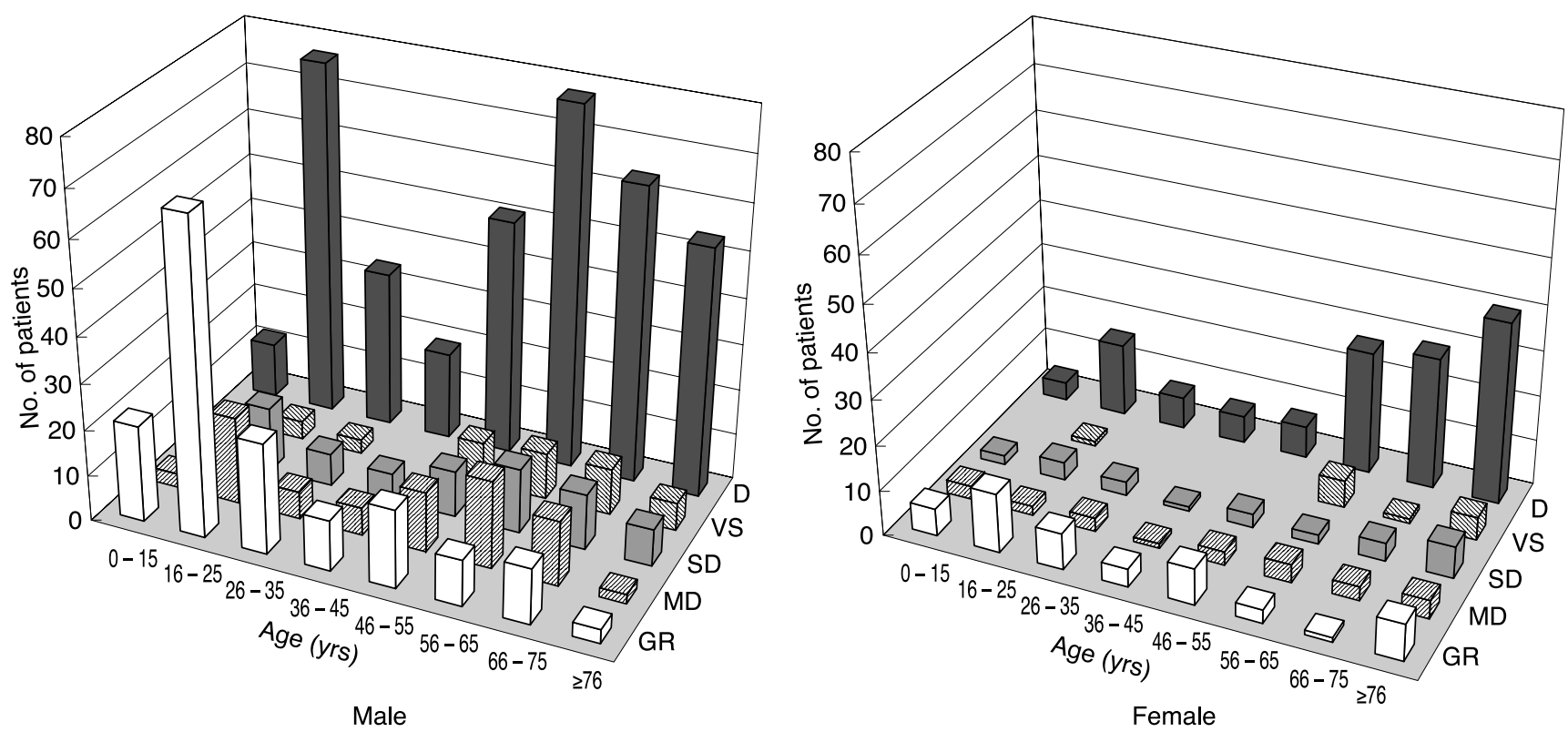

Fig. 6 Final outcome by age and sex. Both deaths (D) and good outcomes (GR) were remarkably high in young males. Both male and female elderly patients had high mortality and very low rates of GR. MD: moderate disability, SD: severe disability, VS: vegetative state.

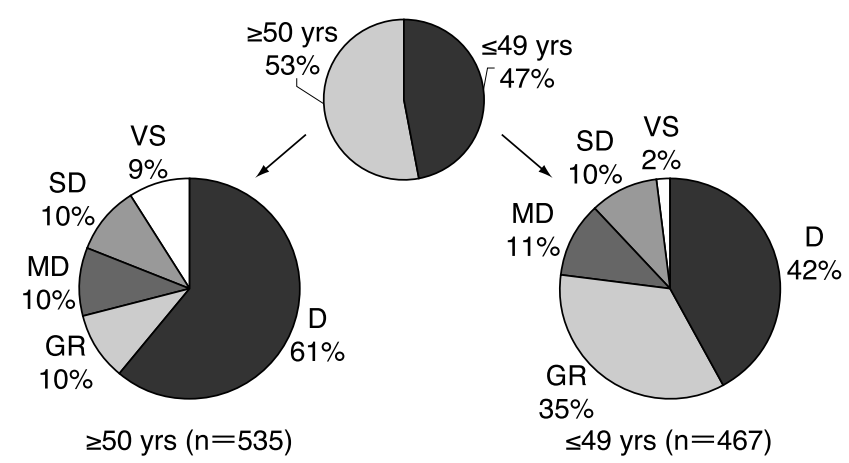

Fig. 7 Glasgow Outcome Scale by age $(n=1,002)$. Mortality was $61 \%$ in patients over 50 years of age and $42 \%$ in patients under 49 years of age. Good recovery (GR) rate was $10 \%$ in the former group and $35 \%$ in the latter. $D$ : dead, MD: moderate disability, SD: severe disability, VS: vegetative state.

injuries seen in highly advanced emergency centers supervised by neurosurgeons. Particular circumstances unique to Japan had to be taken into consideration. Currently, Japan faces a low birth rate, aging population, and injuries pertaining to suicide and child abuse. Our data did not provide information on children under 6 years old, so we could not provide accurate information on child abuse. Also, it was difficult to determine whether a death was caused by falling or suicide without concrete proof of the circumstances. Some of the deaths by falls may have been categorized as accidents when actually they may have been suicides. Classification of head injuries in this area remains ambiguous. The main treatment for traumatic brain injury is the prevention of secondary brain injury, thus the most important factor is pre-hospital care, which includes improvement of ambulance systems and their equipment.

The TCDB in Japan found differences in the causes of injuries, the number of injuries in certain age groups, and the classification of injuries as compared to the TCDB in the U.S.A. For example, the second most common cause of injuries in the U.S.A. is gunshot wounds, which are virtually unknown in Japan. There is also a difference in peak age groups. According to the TCDB in the U.S.A., the number hits a peak in the youth group and begin to decline thereafter, whereas in Japan there are two peaks; one in youth and another in the elderly. ${ }^{7)}$

The two peaks are explicable by social circumstances, such as traffic congestion, density of population, and the ratio of the elderly. However, our present research and data does not take these social factors into consideration. It is only in the future, when a joint study is performed involving nonmedical fields, such as with researchers in motor engineering and traffic science, that the reasons behind the difference in peak age groups between the U.S.A. and Japan can be clarified. 

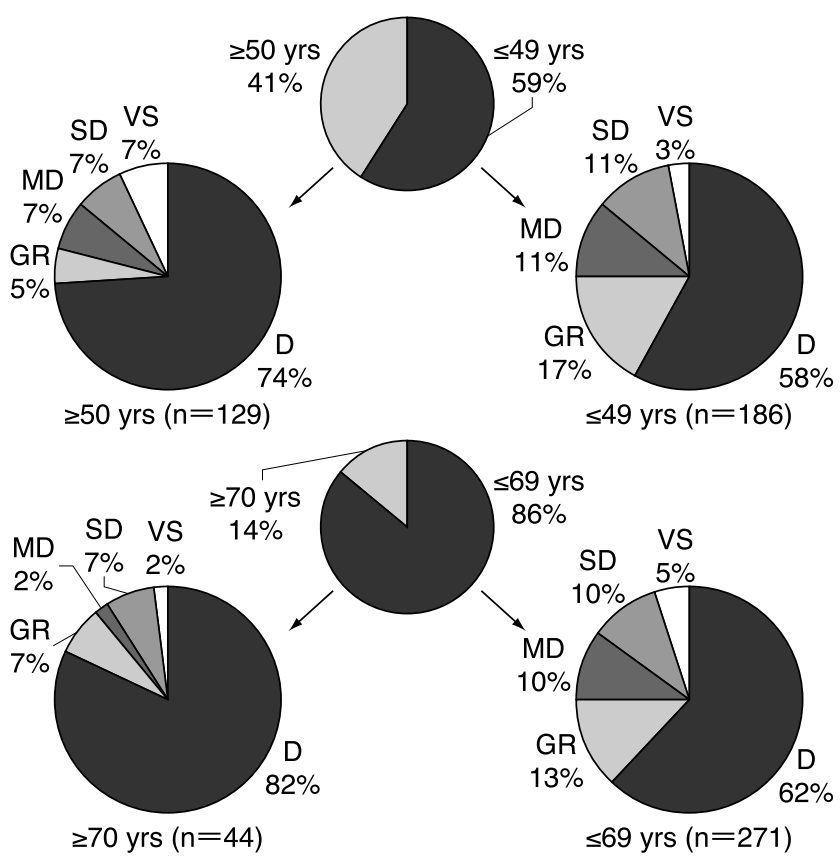

Fig. 8 Outcome of multiple injuries* by age (n = 315). Upper: Mortality was $58 \%$ in patients under 49 years of age and $74 \%$ in patients over 50 years of age. Good recovery (GR) rate was $17 \%$ in the former group and $5 \%$ in the latter. Lower: Mortality was $62 \%$ in patients under 69 years of age and $82 \%$ in patients over 70 years of age. GR rate was $13 \%$ in the former group and $7 \%$ in the latter. D: dead, MD: moderate disability, SD: severe disability, VS: vegetative state. *Cases with Abbreviated Injury Scale 1985 score of 3 or higher in the head/neck and/or other body region.

There are some important points to consider in the treatment of severe head injuries: GCS on arrival at the hospital, age, mechanism of the injury, and other associated injuries. Our studies did not regulate or provide guidance for treatment of traumatic head injuries, but treatment is a necessary factor to determine the outcome. We advised evaluation at 1 month, 3 months, and 6 months after injury, but evaluation at discharge became the final outcome in most cases. The outcome of the injury depends on the GCS evaluation taken within 48 hours of admission, rather than the commonly utilized evaluation of GCS taken immediately on admission.

The occurrence of "talk and deteriorate" (T\&D) cases should especially be emphasized as a factor in the timing of treatment. A series of 721 cases of severe traumatic brain injury included 12\% T\&D cases, and $69 \%$ of them deteriorated within 3 hours. ${ }^{2)}$ This clinical course was seen in many pa- tients over 50 years of age, and frequently in youths with acute epidural hematoma. The incidence of $\mathrm{T} \& \mathrm{D}$ was 1.7 times greater in elderly patients than in youths with acute epidural hematoma, and four times greater in those with cerebral contusion or intracerebral hematoma. Patients with cerebral contusion and intracerebral hematoma showed deterioration within $3-6$ hours in $21 \%$ of cases, and after more than 6 hours in $26 \%$ of cases. CT findings at the time of deterioration showed epidural hematoma with enlarged hematoma, and brain edema or brain swelling with other injuries. The characteristic CT findings of acute epidural hematoma are signs of pathological changes such as cerebral contusion or intracerebral hematoma. The mortality of $\mathrm{T} \& \mathrm{D}$ cases is $52 \%$. Reports on preventable trauma death differ at institutions, and vary in disclosure of information, but we assume that the potential for preventable trauma death is approximately $30 \%$. T\&D is one of the most important neurotraumatological factors that explains the pathogenesis of preventable trauma death, and also explains why GCS at the initial diagnosis is not necessarily indicative of the outcome. It is important, even for specialized neurosurgeons, to create a categorized system for injuries, and for more education in the overall understanding of injuries.

There were 315 cases of multiple injuries with more than two organ injuries of over AIS-85 score 3. Mortality for patients above 50 years of age with multiple injuries was $74 \%$ (under 49 years of age, $58 \%$ ). Furthermore, mortality for patients above 70 years of age $(\mathrm{n}=44)$ was $82 \%$ (under 69 years of age $[\mathrm{n}=271], 62 \%)$. The outcome for elderly patients with multiple injuries was significantly poor.

We had assumed that unified GCS evaluation would be easy to attain, but we found differences in opinions even in the classifications of CT, due to the difficulty in timing of the GCS evaluation, and also in the classifications of traumatic brain injury. Severe brain injuries are especially difficult because the patient's condition is extremely complex, so emphasis should be placed on imaging data such as CT findings. Interpretation of the clinical condition of the patient based on clinical data such as measuring the volume of hematoma is likely to differ from interpretation of CT findings of brain contusion, unilateral brain swelling, or brain edema. The TCDB classifies injuries as either FBI or DBI, but some injuries have both FBI and DBI. Furthermore, we have not unified or established the clinical interpretation of non-evacuated mass due to its complexity and differences in the interpretation at various institutions. In the future, we should begin to record live imaging data for research purposes. 
Our study has attempted to clinically classify traumatic brain injuries including the condition of the injury. The two peaks in the number of injuries found in youth and elderly coincided with data from the National Police Agency and the Ministry of Health, Labor, and Welfare. Also, this study proved that motor vehicle accident injuries increases the number of DBI, and that jumping and falling accidents increases the number of FBI as has been reported in previous studies. CPAOA cases should be studied separately.

The Ministry of Health, Labor, and Welfare is in the process of creating clinical care maps. However, traumatic brain injury has a dynamic and complex character, and constantly changes so the course of treatment is difficult to predict, making it difficult to categorize as a clinical care map as opposed as the present system of payment by results. Traumatic brain injury with complications of multiple injuries is especially difficult to evaluate, but is an important subject to be studied in the future.

This study identified various other important considerations. Patient's personal information must be managed with care to protect their privacy. Furthermore, when examining an unconscious patient, the method of informing and acquiring the patient's consent for the purpose of clinical research need to be discussed. The present state of emergency centers and the evaluation of treatment still have unsolved matters. These issues should be considered for discussion with the "Guidelines for the Treatment and Management of Severe Traumatic Brain Injuries"1) proposed by the Japan Society of Neurotraumatology.

This is the final report of 1,002 cases from the JNTDB describing the general situation of treating severe brain injuries in the acute stage in Japan. We came up with similar results that were presented in the interim report of 721 cases. Some basic problems such as the current state of research on outcome, and the difficulty in categorizing traumatic brain injuries became apparent. Data items must be refined in view of conducting joint research on an international scale.

\section{Acknowledgment}

The main ideas of this article were released at the 27th Japan Society of Neurotraumatology (Tokyo, 2004).

In writing this article we received financial support from The General Insurance Association of Japan, Mitsui Sumitomo Insurance Welfare Foundation, and the Japan Brain Foundation.

\section{References}

1) Committee of Guidelines for the Treatment \& Management of Severe Traumatic Brain Injury: [Guidelines for the Treatment \& Management of Severe Traumatic Brain Injury]. Shinkei Gaisho 23: 1-51, 2000 (Jpn)

2) Kawamata T, Katayama Y: Head injured patients who talk and deteriorate: analysis of 86 cases registered on the Japan Neurotrauma Data Bank. Shinkei Gaisho 25: 205-209, 2002

3) Marshall LF, Becker DP, Bower SA, Cayard C, Eisenberg H, Gross CR, Grossman RG, Jane JA, Kunitz SC, Rimel R, Tabaddor K, Warren J: The National Traumatic Coma Data Bank. Part 1: Design, purpose, goals, and results. J Neurosurg 59: 276-284, 1983

4) Marshall LF, Marshall SB, Klauber MR, van Berkum Clark M, Eisenberg HM, Jane JA, Luerssen TG, Marmarou A, Foulkes MA: A new classification of head injury based on computerized tomography. J Neurosurg 75 (Suppl): S14-20, 1991

5) Ogawa $\mathrm{T}$, Aruga $\mathrm{T}$, Ueda S, Onuma $\mathrm{T}$, Ono J, Katayama Y, Kawamata T, Kandabashi M, Sakamoto T, Shigemori M, Sekino H, Nakamura N, Hirakawa K, Fujimoto M, Marubayashi T, Yamaura A, Yamaoka R, Yamaki T, Yamaguchi T: [Trial for establishment for Japan Neurotrauma Data Bank System]. Shinkei Gaisho 21: 125-129, 1998 (Jpn)

6) Ogawa T, Kawamata T, Sakamoto T, Ono J, Tokutomi $\mathrm{T}$, Nakamura N: [Traumatic coma data bank project in Japan (JTCDB)]. Shinkei Gaisho 25: 117-133, 2002 (Jpn)

7) Vollmer DG, Torner JC, Jane JA, Sadovnic B, Charlebois D, Eisenberg HM, Foulkes MA, Marmarou A, Marshall LF: Age and outcome following traumatic coma: why do older patients fare worse? J Neurosurg 75(Suppl): S37-49, 1991

Address reprint requests to: T. Ogawa, M.D., Department of Emergency Medicine, The Jikei University School of Medicine, 3-25-8 Nishi-Shimbashi, Minato-ku, Tokyo 105-8461, Japan. e-mail: dem@jikei.ac.jp

\section{Commentary}

This four-year study of the Japan Neurotrauma Data Bank is a significant contribution to neurotraumatology. Severe head injury was investigated in an enormous number of more than 1000 comatose patients. Numerous current issues of head injury are highlighted. The causes of head injury in different age groups are revealed to differ considerably. The initial neurological findings proved to be not related with outcome. At 48 hours after the injury, the neurological findings proved to be better related with outcome. This phenomenon is attributed to the high incidence 
of patients who first talk and then deteriorate. This observation was more frequent in the older age group. As the deterioration was noted in $21 \%$ within the first 3 to 6 hours and in 26\% after more than 6 hours, and mortality in these "talk and deteriorate" patients was $52 \%$, one may conclude from this study that the repeat computerized tomography (CT) would be appropriate 6 hours after the injury. Involving 10 hospitals, CT findings had to be classified to arrive at standardized results. The classification in focal brain injury, diffuse brain injury and a combination of both is possibly adequate for CT, but as CT is disclosed to be hopelessly unsatisfactory in depicting focal lesions of the brain stem, which can now be visualized by magnetic resonance imaging, future studies might add magnetic resonance imaging, which currently appears to be better correlated with outcome than CT. The beneficial value of such a study as this cannot be overestimated: Firstly the analysis of the causes may give some clues as to how to improve prevention of head injury, a matter that would probably be far more effective than all neurosurgical efforts combined.
Secondly, in the participating hospitals, the magic of such a study is comparable to a self-fulfilling prophecy. Patients with a head injury will attract proper attention and without any obvious change of treatment results will improve all by themselves. The authors and the participants of this study should be congratulated upon this achievement.

Raimund FIRSCHING, M.D., L.R.C.P., M.R.C.S. Klinik für Neurochirurgie Otto-von-Guericke-Universität Magdeburg, Germany

This is an important paper on the final report from the Japan Neurotrauma Data Bank Project. The data presented in this report is very informative and valuable about traumatic brain injury in Japan that could be useful for not only Japanese but also Asian, European, and American neurosurgeons.

Katsuji SHIma, M.D. Department of Neurosurgery National Defense Medical College Tokorozawa, Saitama, Japan 